\title{
POLYNOMIAL APPROACH FOR THE MOST GENERAL LINEAR FREDHOLM INTEGRODIFFERENTIAL-DIFFERENCE EQUATIONS USING TAYLOR MATRIX METHOD
}

\author{
MEHMET SEZER AND MUSTAFA GÜLSU
}

Received 3 February 2005; Revised 28 March 2006; Accepted 11 May 2006

A Taylor matrix method is developed to find an approximate solution of the most general linear Fredholm integrodifferential-difference equations with variable coefficients under the mixed conditions in terms of Taylor polynomials. This method transforms the given general linear Fredholm integrodifferential-difference equations and the mixed conditions to matrix equations with unknown Taylor coefficients. By means of the obtained matrix equations, the Taylor coefficients can be easily computed. Hence, the finite Taylor series approach is obtained. Also, examples are presented and the results are discussed.

Copyright @ 2006 Hindawi Publishing Corporation. All rights reserved.

\section{Introduction}

An important problem in function theory is the problem of expanding a function in a series of polynomials. Several extensions of the classical theory of Taylor series to differential, differential-difference operators on the real line have shown up recently. Boundaryvalue problems involving integrodifferential-difference equations arise in studying variational problems of control theory where the problem is complicated by the effect of time delays [4, 5], signal transmission [9], biological problems as the problem of determining the expected time for the generation of action potentials in nerve cells by random synaptic inputs in the dendrites [1-3].

Taylor methods to find the approximate solutions of differential equations have been presented in many papers $[6,8,10,11]$. In this paper, the basic ideas of these methods are developed and applied to the high-order general linear differential-difference equation with variable coefficients, which is given in [7, page 229],

$$
\begin{aligned}
& \sum_{k=0}^{m} \sum_{j=0}^{p} P_{k j}(x) y^{(k)}\left(x-\tau_{k j}\right) \\
& \quad=f(x)+\int_{a}^{b} \sum_{i=0}^{q} \sum_{l=0}^{s} K_{i l}(x, t) y^{(i)}\left(t-\tau_{i l}\right) d t, \quad \tau_{k j} \geq 0, \tau_{i l} \geq 0,
\end{aligned}
$$

Hindawi Publishing Corporation International Journal of Mathematics and Mathematical Sciences Volume 2006, Article ID 46376, Pages 1-15

DOI 10.1155/IJMMS/2006/46376 
2 Taylor matrix method

with the mixed conditions

$$
\sum_{k=0}^{m-1} \sum_{r=1}^{R} c_{l k}^{r} y^{(k)}\left(c_{r}\right)=\lambda_{l}, \quad l=1,2, \ldots, m, a \leq c_{r} \leq b
$$

and the solution is expressed as the Taylor polynomial

$$
y(x)=\sum_{n=0}^{N} \frac{y^{(n)}(c)}{n !}(x-c)^{n}, \quad a \leq x, c \leq b .
$$

Here $P_{k j}(x), K_{i l}(x, t)$, and $f(x)$ are functions that have suitable derivatives on $a \leq x, t \leq$ $b$; and $c_{l k}^{r}, c_{r}, c$ and $\tau_{k j}, \tau_{i l}$ are suitable coefficients; $y^{(n)}(c)$ are Taylor coefficients to be determined.

\section{Fundamental matrix relations}

Let us convert the expressions defined in (1.1), (1.2), and (1.3) to matrix forms. We first consider the solution $y(x)$ defined by the truncated Taylor series (1.3) and then we can put it in the matrix form

$$
[y(x)]=\mathbf{X M}_{0} \mathbf{Y}
$$

where,

$$
\begin{gathered}
\mathbf{X}=\left[\begin{array}{lllll}
1 & (x-c) & (x-c)^{2} & \cdots & (x-c)^{N}
\end{array}\right], \\
\mathbf{M}_{0}=\left[\begin{array}{ccccc}
\frac{1}{0 !} & 0 & 0 & \cdots & 0 \\
0 & \frac{1}{1 !} & 0 & \cdots & 0 \\
0 & 0 & \frac{1}{2 !} & \cdots & 0 \\
\vdots & \vdots & \vdots & & \vdots \\
0 & 0 & 0 & \cdots & \frac{1}{N !}
\end{array}\right], \quad \mathbf{Y}=\left[\begin{array}{c}
y^{(0)}(c) \\
y^{(1)}(c) \\
y^{(2)}(c) \\
\vdots \\
y^{(N)}(c)
\end{array}\right] .
\end{gathered}
$$

Now we substitute quantities $\left(x-\tau_{k j}\right)$ instead of $x$ in (1.3) and differentiate it $N$ times with respect to $x$. Then we obtain

$$
\begin{gathered}
y^{(0)}\left(x-\tau_{k j}\right)=\sum_{n=0}^{N} \frac{y^{(n)}(c)}{n !}\left(x-\tau_{k j}-c\right)^{n}, \\
y^{(1)}\left(x-\tau_{k j}\right)=\sum_{n=1}^{N} \frac{y^{(n)}(c)}{(n-1) !}\left(x-\tau_{k j}-c\right)^{n-1},
\end{gathered}
$$




$$
\begin{gathered}
y^{(2)}\left(x-\tau_{k j}\right)=\sum_{n=2}^{N} \frac{y^{(n)}(c)}{(n-2) !}\left(x-\tau_{k j}-c\right)^{n-2}, \\
\vdots \\
y^{(N)}\left(x-\tau_{k j}\right)=\sum_{n=N}^{N} \frac{y^{(n)}(c)}{(n-N) !}\left(x-\tau_{k j}-c\right)^{n-N}
\end{gathered}
$$

and the matrix form, for $x=c$,

$$
\mathbf{Y}\left(\tau_{k j}\right)=\mathbf{X}\left(\tau_{k j}\right) \mathbf{Y}
$$

where

$$
\mathbf{X}\left(\tau_{k j}\right)=\left[\begin{array}{ccccc}
\frac{1}{0 !} & \frac{\left(-\tau_{k j}\right)^{1}}{1 !} & \frac{\left(-\tau_{k j}\right)^{2}}{2 !} & \cdots & \frac{\left(-\tau_{k j}\right)^{N}}{N !} \\
0 & \frac{1}{0 !} & \frac{\left(-\tau_{k j}\right)^{1}}{1 !} & \cdots & \frac{\left(-\tau_{k j}\right)^{N-1}}{(N-1) !} \\
0 & 0 & \frac{1}{0 !} & \cdots & \frac{\left(-\tau_{k j}\right)^{N-2}}{(N-2) !} \\
\vdots & \vdots & \vdots & & \vdots \\
0 & 0 & 0 & \cdots & \frac{1}{0 !}
\end{array}\right], \quad \mathbf{Y}\left(\tau_{k j}\right)=\left[\begin{array}{c}
y^{(0)}\left(c-\tau_{k j}\right) \\
y^{(1)}\left(c-\tau_{k j}\right) \\
y^{(2)}\left(c-\tau_{k j}\right) \\
\vdots \\
y^{(N)}\left(c-\tau_{k j}\right)
\end{array}\right],
$$

On the other hand, we consider terms $P_{k j}(x) y^{(k)}\left(x-\tau_{k j}\right), k=0,1, \ldots, m, j=0,1, \ldots, p$, in (1.1) and can write them as the truncated series expansions of degree $N$ at $x=c$ in the form

$$
P_{k j}(x) y^{(k)}\left(x-\tau_{k j}\right)=\sum_{n=0}^{N} \frac{1}{n !}\left[P_{k j}(x) y^{(k)}\left(x-\tau_{k j}\right)\right]_{x=c}^{(n)}(x-c)^{n} .
$$

By means of Leibnitz's rule we have

$$
\left[P_{k j}(x) y^{(k)}\left(x-\tau_{k j}\right)\right]_{x=c}^{(n)}=\sum_{i=0}^{n}\left(\begin{array}{c}
n \\
i
\end{array}\right) P_{k j}^{(n-i)}(c) y^{(k+i)}\left(c-\tau_{k j}\right)
$$

and substitute in expression (2.6). Thus expression (2.6) becomes

$$
P_{k j}(x) y^{(k)}\left(x-\tau_{k j}\right)=\sum_{n=0}^{N} \sum_{i=0}^{n} \frac{1}{n !}\left(\begin{array}{c}
n \\
i
\end{array}\right) P_{k j}^{(n-i)}(c) y^{(k+i)}\left(c-\tau_{k j}\right)(x-c)^{n}
$$




\section{Taylor matrix method}

and its matrix form

$$
\left[P_{k j}(x) y^{(k)}\left(x-\tau_{k j}\right)\right]=\mathbf{X P}_{k j} \mathbf{Y}\left(\tau_{k j}\right)
$$

or from $(2.4)$

$$
\left[P_{k j}(x) y^{(k)}\left(x-\tau_{k j}\right)\right]=\mathbf{X} \mathbf{P}_{k j} \mathbf{X}\left(\tau_{k j}\right) \mathbf{Y}
$$

where

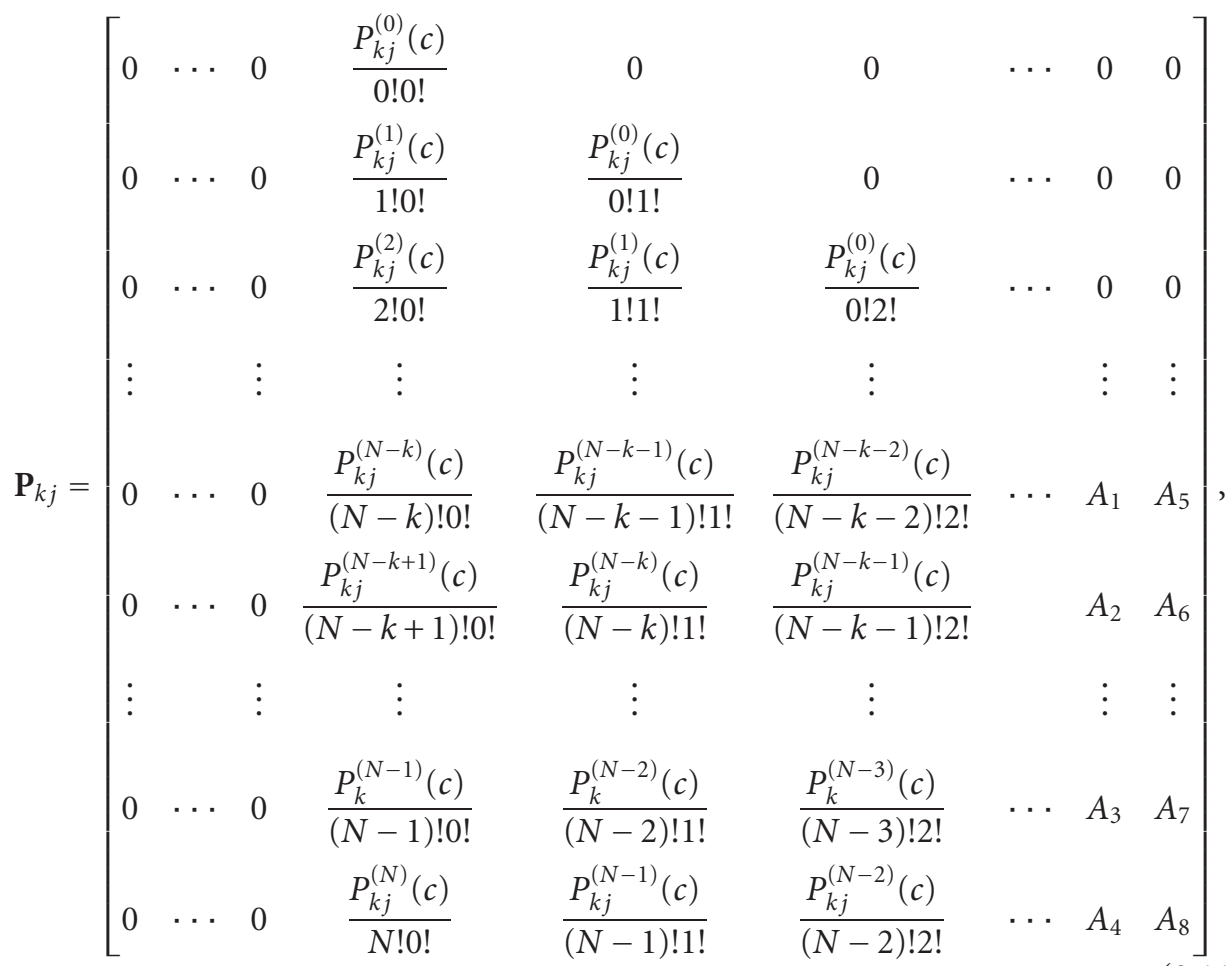

where

$$
\begin{gathered}
A_{1}=\frac{P_{k j}^{(1)}(c)}{1 !(N-k-1) !}, \quad A_{2}=\frac{P_{k j}^{(2)}(c)}{2 !(N-k-1) !}, \quad A_{3}=\frac{P_{k}^{(k)}(c)}{k !(N-k-1) !}, \\
A_{4}=\frac{P_{k j}^{(k+1)}(c)}{(k+1) !(N-k-1) !}, \quad A_{5} \frac{P_{k j}^{(0)}(c)}{0 !(N-k) !}, \quad A_{6}=\frac{P_{k j}^{(1)}(c)}{0 !(N-k) !}, \\
A_{7}=\frac{P_{k}^{(k-1)}(c)}{(k-1) !(N-k) !}, \quad A_{8}=\frac{P_{k j}^{(k)}(c)}{k !(N-k) !} .
\end{gathered}
$$


Let the function $f(x)$ be approximated by a truncated Taylor series

$$
f(x)=\sum_{n=0}^{N} \frac{f^{(n)}(c)}{n !}(x-c)^{n} .
$$

Then we can put this series in the matrix form

$$
[f(x)]=\mathbf{X M}_{0} \mathbf{F}
$$

where the matrices $\mathbf{X}$ and $\mathbf{M}_{0}$ are defined in (2.1); the matrix $\mathbf{F}$ is

$$
\mathbf{F}=\left[\begin{array}{llll}
f^{(0)}(c) & f^{(1)}(c) & \cdots & f^{(N)}(c)
\end{array}\right]^{T}
$$

2.1. Matrix relation for Fredholm integral part. The kernel functions $K_{i l}(x, t),(i=0$, $1, \ldots, q, l=0,1, \ldots, s)$ can be approximated by the truncated Taylor series of degree $N$ about $x=c, t=c$ in the forms

$$
K_{i l}(x, t)=\sum_{n=0}^{N} \sum_{m=0}^{N} k_{n m}^{i l}(x-c)^{n}(t-c)^{m},
$$

where

$$
k_{n m}^{i l}=\frac{1}{n ! m !} \frac{\partial^{n+m} K_{i l}(c, c)}{\partial x^{n} \partial t^{m}}, \quad n, m=0,1, \ldots, N .
$$

The expression (2.16) can be put in the matrix form [6]

$$
\left[\mathbf{K}_{i l}(x, t)\right]=\mathbf{X} \mathbf{K}_{i l} \mathbf{T}^{T},
$$

where

$$
\begin{gathered}
K_{i l}=\left\lfloor k_{n m}^{i l}\right\rfloor, \quad i=0,1, \ldots, q, l=0,1, \ldots, s, \\
\mathbf{T}=\left[\begin{array}{lllll}
1 & (t-c) & (t-c)^{2} & \cdots & (t-c)^{N}
\end{array}\right] .
\end{gathered}
$$

On the other hand, we can obtain the matrix form of the function $y^{(i)}(t)$ as,

$$
\left[y^{(i)}(t)\right]=\mathbf{T M}_{i} \mathbf{Y}, \quad i=0,1, \ldots, q,
$$

and thereby the matrix form of $y^{(i)}\left(t-\tau_{i l}\right)$ as

$$
\left[y^{(i)}\left(t-\tau_{i l}\right)\right]=\mathbf{T}\left(\tau_{i l}\right) \mathbf{M}_{i} \mathbf{Y}, \quad l=0,1, \ldots, s,
$$


where

$$
\begin{aligned}
\mathbf{T}\left(\tau_{i l}\right)= & {\left[\begin{array}{ccccccc}
1 & \left(t-\tau_{i l}-c\right) & \left(t-\tau_{i l}-c\right)^{2} & \cdots & \left(t-\tau_{i l}-c\right)^{N}
\end{array}\right], } \\
M_{i} & =\left[\begin{array}{ccccccc}
0 & 0 & \cdots & \frac{1}{0 !} & 0 & \cdots & 0 \\
0 & 0 & \cdots & 0 & \frac{1}{1 !} & \cdots & 0 \\
\vdots & \vdots & & \vdots & \vdots & & \vdots \\
0 & 0 & \cdots & 0 & 0 & \cdots & \frac{1}{(N-i) !} \\
0 & 0 & \cdots & 0 & 0 & \cdots & 0 \\
\vdots & \vdots & & \vdots & \vdots & & \vdots \\
0 & 0 & \cdots & 0 & 0 & \cdots & 0
\end{array}\right]_{(N+1) x(N+1)}
\end{aligned}
$$

Substituting the matrix forms (2.18) and (2.21) into the integral part of (1.1), we have the matrix relation

$$
\begin{aligned}
{[I(x)] } & =\int_{a}^{b} \sum_{i=0}^{q} \sum_{l=0}^{s} \mathbf{X} \mathbf{K}_{i l} \mathbf{T}^{T} \mathbf{T}\left(\tau_{i l}\right) M_{i} \mathbf{Y} d t \\
& =\mathbf{X} \sum_{i=0}^{q} \sum_{l=0}^{s} \mathbf{K}_{i l}\left\{\int_{a}^{b} \mathbf{T}^{T} \mathbf{T}\left(\tau_{i l}\right) d t\right\} \mathbf{M}_{i} \mathbf{Y}=\mathbf{X} \sum_{i=0}^{q} \sum_{l=0}^{s} \mathbf{K}_{i l} \mathbf{H}_{i l} \mathbf{M}_{i} \mathbf{Y},
\end{aligned}
$$

where

$$
\begin{gathered}
\mathbf{H}_{i l}=\int_{a}^{b} \mathbf{T}^{T} \mathbf{T}\left(\tau_{i l}\right) d t=\left[h_{n m}^{i l}\right], \\
\text { if } \tau_{i l} \neq 0, \quad h_{n m}^{i l}=\sum_{k=0}^{n}\left(\begin{array}{l}
n \\
k
\end{array}\right)\left(\tau_{i l}\right)^{k} \frac{\left(b-\tau_{i l}-c\right)^{n+m-k+1}-\left(a-\tau_{i l}-c\right)^{n+m-k+1}}{n+m-k+1}, \\
\text { if } \tau_{i l}=0, \quad h_{n m}^{i l}=\frac{(b-c)^{n+m+1}-(a-c)^{n+m+1}}{n+m+1}, \quad n, m=0,1, \ldots, N .
\end{gathered}
$$

Substituting the matrix forms (2.10), (2.14), and (2.24) corresponding to the expressions in (1.1) and then simplifying the resulting equation, we have the fundamental matrix equation

$$
\left(\sum_{k=0}^{m} \sum_{j=0}^{p} \mathbf{P}_{k j} \mathbf{X}\left(\tau_{k j}\right)-\sum_{i=0}^{q} \sum_{l=0}^{s} \mathbf{K}_{i l} \mathbf{H}_{i l} \mathbf{M}_{i}\right) \mathbf{Y}=\mathbf{M}_{0} \mathbf{F}, \quad p, q, s<m .
$$

Next, let us form the matrix representation for the conditions (1.2) as follows [6].

The expression (1.3) and its derivatives are equivalent to the matrix equations

$$
\left[y^{(k)}(x)\right]=\mathbf{X M}_{k} \mathbf{Y}, \quad k=0,1, \ldots, m-1,
$$


where the matrix $M_{k}$ is defined in the expressions (2.21). By using these equations, the quantities $y^{(k)}\left(c_{r}\right), k=0,1, \ldots, m-1, r=1,2, \ldots, R, a \leq c_{r} \leq b$, can be written as

$$
\left[y^{(k)}\left(c_{r}\right)\right]=\mathbf{C}_{r} \mathbf{M}_{k} \mathbf{Y}
$$

where

$$
\mathbf{C}_{r}=\left[\begin{array}{lllll}
1 & \left(c_{r}-c\right) & \left(c_{r}-c\right)^{2} & \cdots & \left(c_{r}-c\right)^{N}
\end{array}\right]
$$

Substituting quantities (2.28) into (1.2) and then simplifying, we obtain the matrix forms corresponding to the $m$ mixed conditions as

$$
\mathbf{U}_{l} \mathbf{Y}=\left[\lambda_{l}\right], \quad l=1,2, \ldots, m,
$$

where

$$
\mathbf{U}_{l}=\sum_{k=0}^{m-1} \sum_{r=1}^{R} c_{l k}^{r} \mathbf{C}_{r} \mathbf{M}_{k} \equiv\left[\begin{array}{llll}
u_{l 0} & u_{l 1} & \cdots & u_{l n}
\end{array}\right]
$$

and the constants $\mathcal{u}_{l n}, n=0,1, \ldots, N, l=1,2, \ldots, m$, are related to the coefficients $c_{l k}^{r}$ and $c_{r}$.

\section{Method of solution}

The fundamental matrix equation (2.26) for the high-order general linear Fredholm integrodifferential-difference equation with variable coefficients corresponds to a system of $(N+1)$ algebraic equations for the $(N+1)$ unknown coefficients $y^{(0)}(c), y^{(1)}(c), \ldots$, $y^{(N)}(c)$.

Briefly we can write (2.26) in the form

$$
\mathbf{W Y}=\mathbf{M}_{0} \mathbf{F} \text { or }\left[\mathbf{W} ; \mathbf{M}_{0} \mathbf{F}\right] \text {, }
$$

where

$$
\mathbf{W}=\left[w_{h n}\right]=\left(\sum_{k=0}^{m} \sum_{j=0}^{p} \mathbf{P}_{k j} \mathbf{X}\left(\tau_{k j}\right)-\sum_{i=0}^{q} \sum_{l=0}^{s} \mathbf{K}_{i l} \mathbf{H}_{i l} \mathbf{M}_{i}\right), \quad h, n=0,1, \ldots, N .
$$

The augmented matrix of (3.1) becomes

$$
\left[\mathbf{W} ; \mathbf{M}_{0} \mathbf{F}\right]=\left[\begin{array}{cccccc}
w_{00} & w_{01} & \cdots & w_{0 N} & ; & \frac{f^{(0)}(c)}{0 !} \\
w_{10} & w_{11} & \cdots & w_{1 N} & ; & \frac{f^{(1)}(c)}{1 !} \\
\vdots & \vdots & & \vdots & \vdots \\
w_{N 0} & w_{N 1} & \cdots & w_{N N} & ; & \frac{f^{(N)}(c)}{N !}
\end{array}\right] .
$$


Consequently, to find the unknown Taylor coefficients $y^{(n)}(c), n=0(1) N$, related with the approximate solution of the problem consisting of (1.1) and conditions (1.2), by replacing the $m$ row matrices (2.30) by the last $m$ rows of augmented matrix (3.3), we have a new augmented matrix

$$
\left[\mathbf{W}^{*} ; \mathbf{F}^{*}\right]=\left[\begin{array}{cccccc}
w_{00} & w_{01} & \cdots & w_{0 N} & ; & \frac{f^{(0)}(c)}{0 !} \\
w_{10} & w_{11} & \cdots & w_{1 N} & ; & \frac{f^{(1)}(c)}{1 !} \\
\cdots & \cdots & & \cdots & ; & \cdots \\
w_{N-m, 0} & w_{N-m, 1} & \cdots & w_{N-m, N} & ; & \frac{f^{(N-m)}(c)}{(N-m) !} \\
u_{00} & u_{01} & \cdots & u_{0 N} & ; & \mu_{0} \\
u_{10} & u_{11} & \cdots & u_{1 N} & ; & \mu_{1} \\
\cdots & \cdots & & \cdots & ; & \cdots \\
u_{m-1,0} & u_{m-1,1} & \cdots & u_{m-1, N} & ; & \mu_{m-1}
\end{array}\right]
$$

or the corresponding matrix equation

$$
\mathbf{W}^{*} \mathbf{Y}=\mathbf{F}^{*}
$$

If $\operatorname{det} \mathbf{W}^{*} \neq 0$, we can write $(2.14)$ as

$$
\mathbf{Y}=\left(\mathbf{W}^{*}\right)^{-1} \mathbf{F}^{*}
$$

and the matrix $\mathbf{Y}$ is uniquely determined. Thus our problem has a unique solution. This solution is given by the truncated Taylor series (1.3).

Also we can easily check the accuracy of the obtained solutions as follows $[6,8]$.

Since the Taylor polynomial (1.3) is an approximate solution of (1.1), when the function $y(x)$ and its derivatives are substituted in (1.1), the resulting equation must be satisfied approximately; that is, for $x=x_{i} \in[a, b], i=0,1,2, \ldots$,

$$
D\left(x_{i}\right)=\left|\sum_{k=0}^{m} \sum_{j=0}^{p} P_{k j}(x) y^{(k)}\left(x-\tau_{k j}\right)-\int_{a}^{b} \sum_{i=0}^{q} \sum_{l=0}^{s} K_{i l}(x, t) y^{(i)}\left(t-\tau_{i l}\right) d t-f(x)\right| \cong 0
$$

or

$$
D\left(x_{i}\right) \leq 10^{-k_{i}} \quad\left(k_{i} \text { is any positive integer }\right)
$$

If $\max \left|10^{-k_{i}}\right|=10^{-i}$ ( $i$ is any positive integer) is prescribed, then the truncation limit $N$ is increased until the difference $D\left(x_{i}\right)$ at each of the points becomes smaller than the prescribed $10^{-i}$. 


\section{Illustrations}

The method of this study is useful in finding the solutions of general linear Fredholm integrodifferential-difference equations in terms of Taylor polynomials. We illustrate it by the following examples.

Example 4.1. Let us first consider the third-order linear Fredholm integrodifferentialdifference equation

$$
y^{\prime \prime \prime}(x)-x y^{\prime \prime}\left(x-\frac{\pi}{2}\right)-y^{\prime}(x-\pi)=x \sin (x)+\int_{-\pi / 2}^{\pi / 2}\left[x y^{\prime}(t)-t y(t)+t y^{\prime \prime}(t-\pi)\right] d t
$$

with the conditions

$$
y(0)=1, \quad y^{\prime}(0)=0, \quad y^{\prime \prime}(0)=-1,
$$

and approximate the solution $y(x)$ by the polynomial

$$
y(x)=\sum_{n=0}^{6} \frac{y^{(n)}(0)}{n !}(x-c)^{n}
$$

where $P_{00}(x)=0, P_{10}(x)=-1, P_{20}(x)=-x, P_{30}(x)=1, \tau_{00}=0, \tau_{10}=\pi, \tau_{20}=\pi / 2, \tau_{30}=$ $0,-\pi / 2 \leq x \leq \pi / 2, c=0, N=6, p=1, m=3, f(x)=x \sin (x)$.

We first reduce this equation, from (2.26) to the matrix form

$$
\left\{\sum_{k=0}^{3} \sum_{j=0}^{0} \mathbf{P}_{k j} \mathbf{X}\left(\tau_{k j}\right)-\sum_{i=0}^{2} \sum_{l=0}^{0} \mathbf{K}_{i l} \mathbf{H}_{i l} \mathbf{M}_{i}\right\} \mathbf{Y}=\mathbf{M}_{0} \mathbf{F}
$$

or clearly

$$
\begin{aligned}
& \left\{\left(P_{00} X\left(\tau_{00}\right)+P_{10} X\left(\tau_{10}\right)+P_{20} X\left(\tau_{20}\right)+P_{30} X\left(\tau_{30}\right)\right)\right. \\
& \left.-\left(K_{00} H_{00} M_{0}+K_{10} H_{10} M_{1}+K_{20} H_{20} M_{2}\right)\right\} \mathbf{Y}=\mathbf{M}_{0} \mathbf{F} .
\end{aligned}
$$

From (2.30), the matrices for conditions are computed as

$$
\begin{array}{rlrl}
\mathbf{U}_{0} & =\left[\begin{array}{lllllll}
1 & 0 & 0 & 0 & 0 & 0 & 0
\end{array}\right], & \lambda_{0}=1, \\
\mathbf{U}_{1}=\left[\begin{array}{lllllll}
0 & 1 & 0 & 0 & 0 & 0 & 0
\end{array}\right], & \lambda_{1}=0, \\
\mathbf{U}_{2}=\left[\begin{array}{lllllll}
0 & 0 & 1 & 0 & 0 & 0 & 0
\end{array}\right], & \lambda_{2}=-1 .
\end{array}
$$


Substituting the above matrices into the fundamental matrix equation and using the simple computations, we have the augmented matrix based on conditions which is

$$
\left[\mathbf{W}^{*} ; \mathbf{F}^{*}\right]=\left[\begin{array}{cccccccccc}
0 & -1+\frac{\pi^{3}}{12} & \pi & \frac{-\pi^{2}}{2}+1+\frac{\pi^{5}}{480}-\frac{\pi^{3}}{12} & \frac{\pi^{3}}{6}+\frac{\pi^{4}}{12} & B_{1} & B_{5} & ; & 0 \\
0 & -\pi & -2 & \frac{3 \pi}{2}-\frac{\pi^{3}}{24} & \frac{-5 \pi^{2}}{8}+1 & B_{2} & B_{6} & ; & 0 \\
0 & 0 & 0 & \frac{-3}{2} & \pi & B_{3} & B_{7} & ; & 1 \\
0 & 0 & 0 & 0 & \frac{-2}{3} & B_{4} & B_{8} & ; & 0 \\
1 & 0 & 0 & 0 & 0 & 0 & 0 & ; & 1 \\
0 & 1 & 0 & 0 & 0 & 0 & 0 & 0 & ; & 0 \\
0 & 0 & 1 & 0 & 0 & 0 & ; & -1
\end{array}\right] \text {, }
$$

where

$$
\begin{gathered}
B_{1}=\frac{-\pi^{4}}{24}+\frac{\pi^{7}}{53760}-\frac{\pi^{5}}{160}, \quad B_{2}=\frac{3 \pi^{3}}{16}-\frac{\pi^{5}}{1920}, \quad B_{3}=\frac{-3 \pi^{2}}{8}+\frac{1}{2}, \\
B_{4}=\frac{5 \pi}{12}, \quad B_{5}=\frac{\pi^{5}}{120}+\frac{23 \pi^{6}}{1440}, \quad B_{6}=\frac{-17 \pi^{4}}{384}, \\
B_{7}=\frac{5 \pi^{3}}{48}, \quad B_{8}=\frac{-7 \pi^{2}}{48}+\frac{1}{6} .
\end{gathered}
$$

Solving this system, Taylor coefficients are obtained as $y^{(0)}(0)=1, y^{(1)}(0)=0, y^{(2)}(0)=$ $-1, y^{(3)}(0)=-0.2181409971, y^{(4)}(0)=0.4585894230, y^{(5)}(0)=0.06597637009, y^{(2)}(0)=$ -0.1723673475 .

Thereby the solution of the given problem under the condition (1.2) becomes

$$
\begin{aligned}
y(x)= & 1-\frac{1}{2} x^{2}-0.036356832 x^{3}+0.019107892 x^{4} \\
& +0.00054980308 x^{5}-0.00023939909 x^{6} .
\end{aligned}
$$

We use the absolute error to measure the difference between the numerical and exact solutions. In Table 4.1 the errors obtained for $N=6,7,8$ are given with the exact solution $y(x)=\cos (x)$. 
Table 4.1. Error analysis of Example 4.1 for the $x$ value.

\begin{tabular}{ccccc}
\hline$x$ & $\begin{array}{c}\text { Exact } \\
\text { solution }\end{array}$ & $\begin{array}{c}N=6 \\
E\left(x_{i}\right)\end{array}$ & $\begin{array}{c}N=7 \\
E\left(x_{i}\right)\end{array}$ & $\begin{array}{c}N=8 \\
E\left(x_{i}\right)\end{array}$ \\
\hline$-\pi / 2$ & 0.000000 & 0.256620 & 0.102547 & 0.015526 \\
$-2 \pi / 5$ & 0.309016 & 0.122302 & 0.049204 & 0.008451 \\
$-3 \pi / 10$ & 0.587785 & 0.047036 & 0.019549 & 0.003843 \\
$-\pi / 5$ & 0.809016 & 0.012410 & 0.005450 & 0.001241 \\
$-\pi / 10$ & 0.951056 & 0.001344 & 0.000637 & 0.000169 \\
0 & 1.000000 & 0.000000 & 0.000000 & 0.000000 \\
$\pi / 10$ & 0.951056 & 0.000906 & 0.000541 & 0.000201 \\
$\pi / 5$ & 0.809016 & 0.005518 & 0.003914 & 0.001728 \\
$3 \pi / 10$ & 0.587785 & 0.013019 & 0.011765 & 0.006151 \\
$2 \pi / 5$ & 0.309016 & 0.018544 & 0.024459 & 0.015087 \\
$\pi / 2$ & 0.000000 & 0.014686 & 0.041279 & 0.029890 \\
\hline
\end{tabular}

Example 4.2. Second we can study the following first-order linear differential-difference equation with variable coefficients:

$$
\begin{aligned}
y^{\prime \prime \prime}(x) & -x y^{\prime \prime}\left(x-\frac{\pi}{2}\right)+y\left(x+\frac{\pi}{2}\right) \\
& =2-x \cos (x)+\int_{-\pi / 2}^{\pi / 2}\left[x y^{\prime}(t)-t y(t)+t y^{\prime \prime}\left(t+\frac{\pi}{2}\right)+x y\left(t-\frac{\pi}{2}\right)\right] d t
\end{aligned}
$$

with the conditions

$$
y(0)=0, \quad y^{\prime}(0)=1, \quad y^{\prime \prime}(0)=0,
$$

and approximate the solution $y(x)$ by the polynomial

$$
y(x)=\sum_{n=0}^{5} \frac{y^{(n)}(0)}{n !}(x-c)^{n},
$$

where $-\pi / 2 \leq x, t \leq \pi / 2, \lambda=1, \mu=1, m=3, N=5, P_{00}(x)=1, P_{20}(x)=-x, P_{30}(x)=1$, $\tau_{00}=-\pi / 2, \tau_{01}=2, \tau_{20}=\pi / 2, \tau_{30}=0, K_{00}(x, t)=-t, K_{01}(x, t)=x, K_{10}(x, t)=x, K_{20}(x$, $t)=\pi / 2, c=0, N=6, m=1, p=1, \tau_{00}^{*}=0, \tau_{01}^{*}=\pi / 2, \tau_{10}^{*}=0, \tau_{20}^{*}=\pi / 2, f(x)=2-$ $x \cos (x)$.

Then for $N=5$, the fundamental matrix equation from (2.26) becomes

$$
\left\{\sum_{k=0}^{3} \sum_{j=0}^{0} \mathbf{P}_{k j} \mathbf{X}\left(\tau_{k j}\right)-\sum_{i=0}^{2} \sum_{l=0}^{1} \mathbf{K}_{i l} \mathbf{H}_{i l} \mathbf{M}_{i}\right\} \mathbf{Y}=\mathbf{M}_{0} \mathbf{F} .
$$


Following the previous procedures, the augmented matrix $\left[\mathbf{W}^{*} ; \mathbf{F}^{*}\right]$ based on the conditions is obtained as

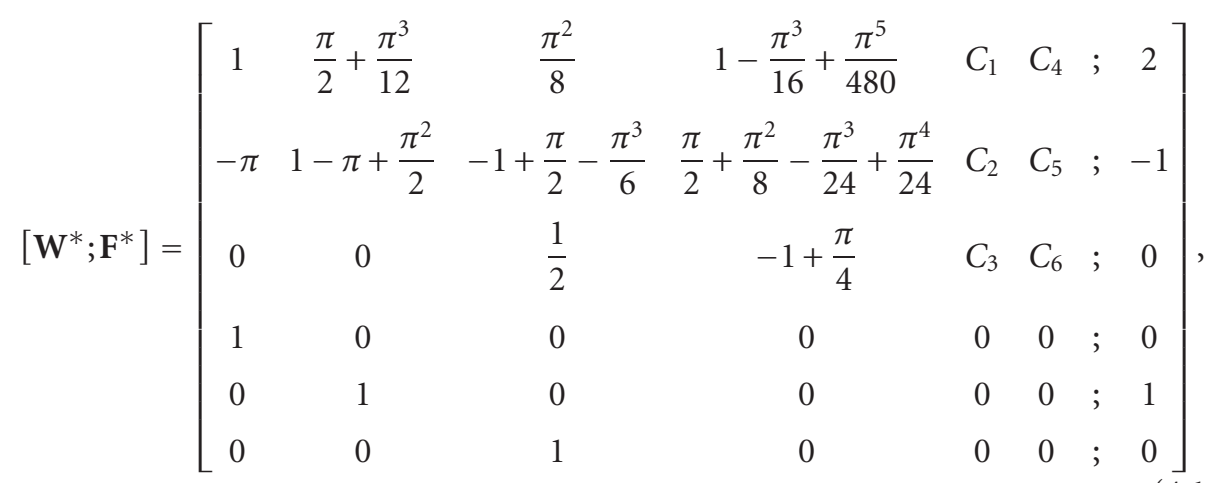

where

$$
\begin{gathered}
C_{1}=\frac{-5 \pi^{4}}{128}, \quad C_{2}=1-\frac{\pi^{2}}{8}+\frac{\pi^{3}}{48}-\frac{\pi^{5}}{120}, \quad C_{3}=\frac{\pi}{2}+\frac{\pi^{2}}{16}, \\
C_{4}=\frac{-47 \pi^{5}}{3840}+\frac{\pi^{7}}{53760}, \quad C_{5}=\frac{\pi^{3}}{48}+\frac{\pi^{4}}{384}-\frac{\pi^{5}}{1920}+\frac{\pi^{6}}{720}, \quad C_{6}=\frac{1}{2}-\frac{\pi^{2}}{8}+\frac{\pi^{3}}{96} .
\end{gathered}
$$

Solving this system, Taylor coefficients are obtained as $y^{(0)}(0)=0, y^{(1)}(0)=1, y^{(2)}(0)=0$, $y^{(3)}(0)=-0.9034070707, y^{(4)}(0)=0.02918158630, y^{(5)}(0)=0.6274658043$.

Thereby the solution of the given problem under the condition (1.2) becomes

$$
y(x)=x-0.1505678451 x^{3}+0.001215899429 x^{4}+0.005228881702 x^{5} .
$$

We use the absolute error to measure the difference between the numerical and exact solutions. In Table 4.2 the solutions obtained for $N=5,7$ are compared with the exact solution $y(x)=\sin (x)$.

Example 4.3. Our last example is the second-order linear differential-difference equation

$$
y^{\prime \prime}(x)-x y^{\prime}(x-1)+y(x-2)=-x^{2}-2 x+5
$$

with the conditions

$$
y(0)=-1, \quad y^{\prime}(-1)=-2, \quad-2 \leq x \leq 0,
$$

and approximate the solution $y(x)$ by the polynomial

$$
y(x)=\sum_{n=0}^{7} \frac{y^{(n)}(0)}{n !}(x-c)^{n},
$$


Table 4.2. Error analysis of Example 4.2 for the $x$ value.

\begin{tabular}{|c|c|c|c|c|c|}
\hline \multirow[t]{2}{*}{$x$} & \multicolumn{3}{|c|}{$N=5$} & \multicolumn{2}{|l|}{$N=7$} \\
\hline & Exact & Num. Sol. & $E\left(x_{i}\right)$ & Num. Sol. & $E\left(x_{i}\right)$ \\
\hline$-\pi / 2$ & -1.000000 & -1.029829 & 0.029829 & -1.005851 & 0.005851 \\
\hline$-2 \pi / 5$ & -0.951056 & -0.971203 & 0.020146 & -0.955063 & 0.004006 \\
\hline$-3 \pi / 10$ & -0.809016 & -0.819355 & 0.010338 & -0.811113 & 0.002096 \\
\hline$-\pi / 5$ & -0.587785 & -0.591292 & 0.003507 & -0.588510 & 0.000724 \\
\hline$-\pi / 10$ & -0.309016 & -0.309494 & 0.000477 & -0.309117 & 0.000100 \\
\hline 0 & 0.000000 & 0.000000 & 0.000000 & 0.000000 & 0.000000 \\
\hline$\pi / 10$ & 0.309016 & 0.309518 & 0.000501 & 0.309125 & 0.000108 \\
\hline$\pi / 5$ & 0.587785 & 0.591671 & 0.003886 & 0.588632 & 0.000847 \\
\hline $3 \pi / 10$ & 0.809016 & 0.821274 & 0.012257 & 0.811726 & 0.002709 \\
\hline $2 \pi / 5$ & 0.951056 & 0.977267 & 0.026210 & 0.956973 & 0.005916 \\
\hline$\pi / 2$ & 1.000000 & 1.044634 & 0.044634 & 1.010431 & 0.010431 \\
\hline
\end{tabular}

where $-2 \leq x \leq 0, m=2, p=1, c=0, N=7, P_{00}(x)=1, P_{10}(x)=-x, P_{20}(x)=1, \tau_{00}=$ 2, $\tau_{10}=1, \tau_{20}=0, f(x)=-x^{2}-2 x+5$.

Then for $N=7$, the fundamental matrix equation from (2.26) becomes

$$
\sum_{k=0}^{2} \sum_{j=0}^{0} \mathbf{P}_{k j} \mathbf{X}\left(\tau_{k j}\right) \mathbf{Y}=\mathbf{M}_{0} \mathbf{F} .
$$

To find a Taylor polynomial solution of the problem above, we first take $c=0, N=7$ and then proceed as before. Then we obtain the desired augmented matrix

$$
\left[\mathbf{W}^{*} ; \mathbf{F}^{*}\right]=\left[\begin{array}{cccccccccc}
1 & -2 & 3 & -\frac{4}{3} & \frac{2}{3} & -\frac{4}{15} & \frac{4}{45} & -\frac{8}{315} & ; & 5 \\
0 & 0 & -1 & \frac{5}{2} & -\frac{7}{6} & \frac{5}{8} & -\frac{31}{120} & \frac{7}{80} & ; & -2 \\
0 & 0 & -\frac{1}{2} & 0 & 1 & -\frac{1}{2} & \frac{7}{24} & -\frac{1}{8} & ; & -1 \\
0 & 0 & 0 & -\frac{1}{3} & \frac{1}{6} & \frac{1}{4} & -\frac{5}{36} & \frac{13}{144} & ; & 0 \\
0 & 0 & 0 & 0 & -\frac{1}{8} & \frac{1}{12} & \frac{1}{24} & -\frac{1}{36} & ; & 0 \\
0 & 0 & 0 & 0 & 0 & -\frac{1}{30} & \frac{1}{40} & \frac{1}{240} & ; & 0 \\
1 & 0 & 0 & 0 & 0 & 0 & 0 & 0 & ; & -1 \\
0 & 1 & -1 & \frac{1}{2} & -\frac{1}{6} & \frac{1}{24} & -\frac{1}{120} & \frac{1}{720} & ; & -2
\end{array}\right] .
$$


From the solution of this system, the coefficients $y^{(n)}(0)(n=0,1, \ldots, 7)$ are uniquely determined as

$$
\mathbf{Y}=\left[\begin{array}{lllllll}
-1 & 0 & 2 & 0 & 0 & 0 & 0
\end{array}\right]^{T}
$$

By substituting the obtained coefficients (4.22) the solution of (4.17) becomes

$$
y(x)=x^{2}-1
$$

which is an exact solution.

\section{Conclusions}

The method presented in this study is useful in finding approximate and also exact solutions of general linear Fredholm integrodifferential-difference equations. Equation (1.1) can be reduced to differential equations, difference equations, integral equations, integrodifferential equations, and also the given method can be applied to all these equations.

Differential-difference equations with variable coefficients are usually difficult to be solved analytically. In this case, the presented method is required for the approximate solutions. On the other hand, it is observed that the method has the best advantage when the known functions in equation can be expanded to Taylor series with rapid convergence. In addition, an interesting feature of this method is to find the analytical solutions if the equation has an exact solution that is a polynomial of degree $N$ or less than $N$.

A considerable advantage of the method is that Taylor coefficients of the solution are found very easily by using the computer programs. We can use the symbolic algebra program, Maple, to find the Taylor coefficients of the solution.

Also, the method can be developed and applied to system of linear integrodifferentialdifference equations, but some modifications are required.

\section{Acknowledgments}

The authors thank the anonymous referees for their very valuable discussions and suggestions, which led to a great improvement of the paper.

\section{References}

[1] D. D. Bainov, M. B. Dimitrova, and A. B. Dishliev, Oscillation of the bounded solutions of impulsive differential-difference equations of second order, Applied Mathematics and Computation 114 (2000), no. 1, 61-68.

[2] J. Cao and J. Wang, Delay-dependent robust stability of uncertain nonlinear systems with time delay, Applied Mathematics and Computation 154 (2004), no. 1, 289-297.

[3] Z. S. Hu, Boundedness of solutions to functional integro-differential equations, Proceedings of the American Mathematical Society 114 (1992), no. 2, 519-526.

[4] M. K. Kadalbajoo and K. K. Sharma, Numerical analysis of boundary-value problems for singularly-perturbed differential-difference equations with small shifts of mixed type, Journal of Optimization Theory and Applications 115 (2002), no. 1, 145-163.

[5] _ Numerical analysis of singularly perturbed delay differential equations with layer behavior, Applied Mathematics and Computation 157 (2004), no. 1, 11-28. 
[6] R. P. Kanwal and K. C. Liu, A Taylor expansion approach for solving integral equation, International Journal of Mathematical Education in Science and Technology 20 (1989), no. 3, 411-414.

[7] H. Levy and F. Lessman, Finite Difference Equations, The Macmillan, New York, 1961.

[8] Ş. Nas, S. Yalçınbaş, and M. Sezer, A Taylor polynomial approach for solving high-order linear Fredholm integro-differential equations, International Journal of Mathematical Education in Science and Technology 31 (2000), no. 2, 213-225.

[9] T. L. Saaty, Modern Nonlinear Equations, Dover, New York, 1981.

[10] M. Sezer, Taylor polynomial solutions of Volterra integral equations, International Journal of Mathematical Education in Science and Technology 25 (1994), no. 5, 625-633.

[11] _ A method for approximate solution of the second order linear differential equations in terms of Taylor polynomials, International Journal of Mathematical Education in Science and Technology 27 (1996), no. 6, 821-834.

Mehmet Sezer: Department of Mathematics, Faculty of Science, Mugla University, 48000 Mugla, Turkey

E-mail address: msezer@mu.edu.tr

Mustafa Gülsu: Department of Mathematics, Faculty of Science, Mugla University, 48000 Mugla, Turkey

E-mail address: mgulsu@mu.edu.tr 


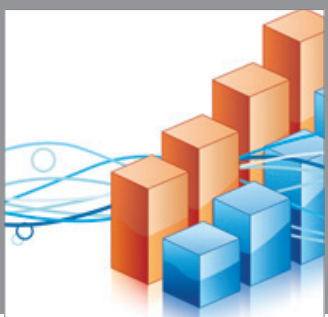

Advances in

Operations Research

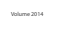

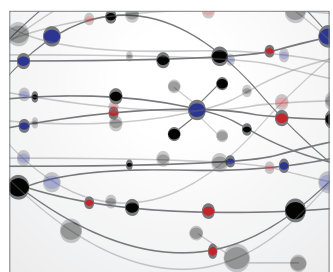

\section{The Scientific} World Journal
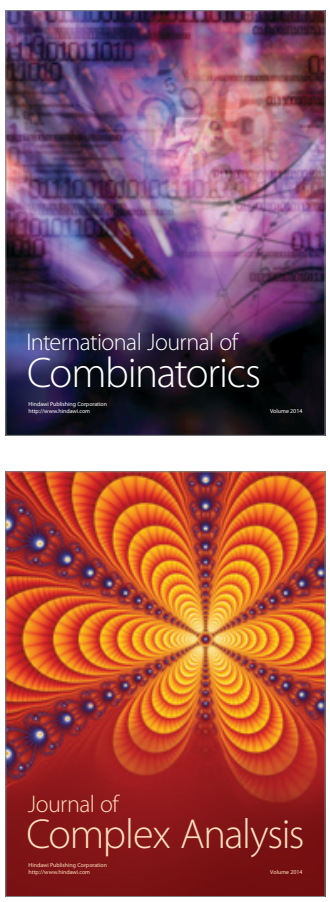

International Journal of

Mathematics and

Mathematical

Sciences
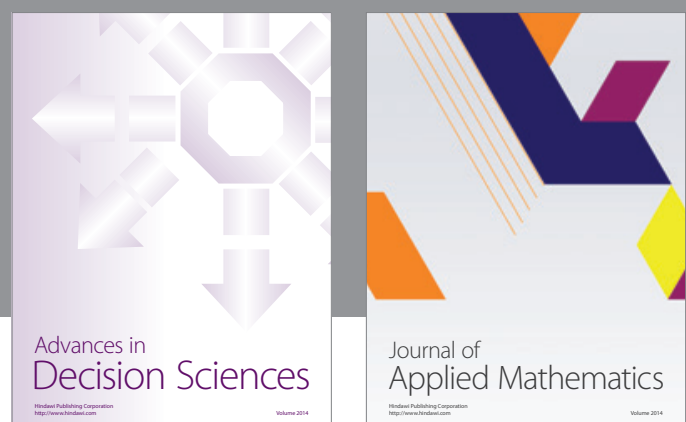

Journal of

Applied Mathematics
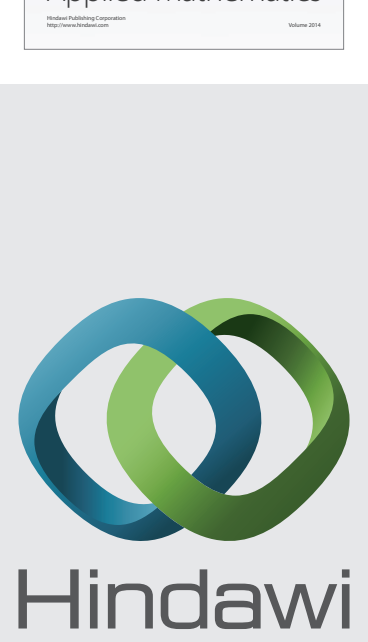

Submit your manuscripts at http://www.hindawi.com
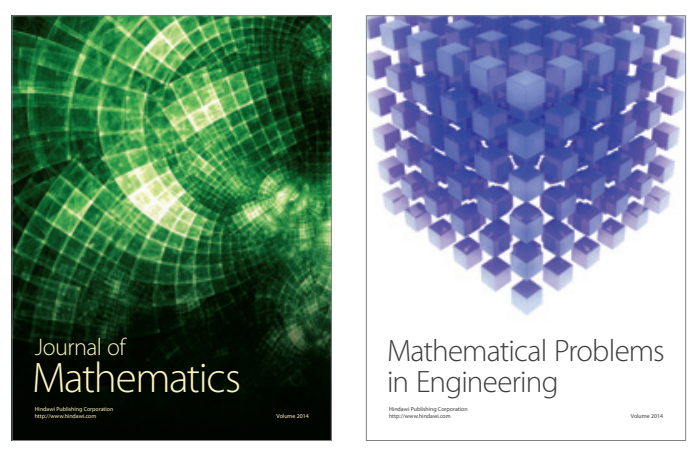

Mathematical Problems in Engineering
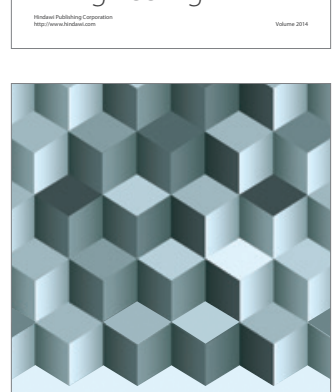

Journal of

Function Spaces
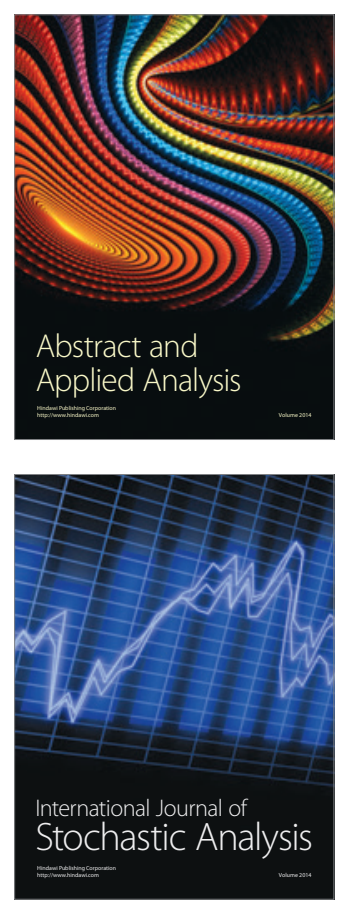

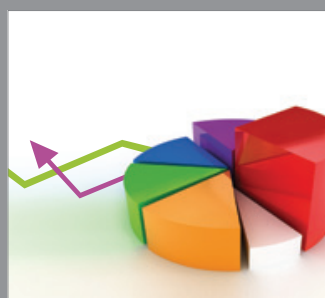

ournal of

Probability and Statistics

Promensencen
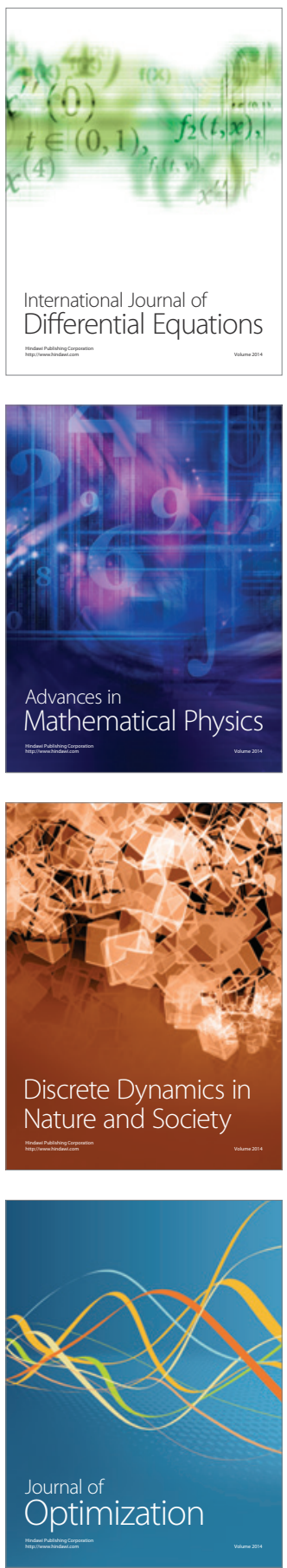